\title{
Publisher's Note: Probing weak dipole-dipole interaction using phase-modulated nonlinear spectroscopy [Phys. Rev. A 95, 052509 (2017)]
}

Zeng-Zhao Li, Lukas Bruder, Frank Stienkemeier, and Alexander Eisfeld

Q (Received 21 June 2019; published 28 June 2019)

DOI: 10.1103/PhysRevA.99.069902

This paper was published online on 26 May 2017 with an omission of an acknowledgment to a funding source. The acknowledgment should read as "This work was supported by the European Research Council (ERC) H2020 funding Advanced Grant COCONIS (694965).” The acknowledgment has been added as of 24 June 2019. The acknowledgment is not present in the printed version of the journal. 